\title{
Caregiver Burden is Reduced by Personalized Social Support for Non-Dependent Old Persons: a Longitudinal Study of 876 Old Persons and Their Caregivers
}

Sylvie Arlotto ( $\nabla$ sylvie.arlotto@ap-hm.fr)

Assistance Publique Hopitaux de Marseille https://orcid.org/0000-0002-2548-8001

Stéphanie Gentile

Aix-Marseille Universite

Anne Claire Durand

Assistance Publique Hopitaux de Marseille

Sylvie Bonin-Guillaume

Aix-Marseille Universite

Research article

Keywords: Caregiver's burden, Old person, Personalized social support

Posted Date: May 8th, 2020

DOI: https://doi.org/10.21203/rs.2.18570/v3

License: (c) (1) This work is licensed under a Creative Commons Attribution 4.0 International License.

Read Full License 


\section{Abstract}

Background. Informal care provided by family caregivers to old persons is associated with a high risk of burden and poor health status. This study aimed to analyze the impact of Personalized Social Support (PSS) for non-dependent old persons living in the community on caregiver burden, satisfaction, and frailty.

Methods . This non-interventional longitudinal study was performed in the southeast of France: old persons asking for PSS ( $>70$ years of age, with no disability and no severe chronic disease, living at home) and their caregivers were included with a 6-month follow-up. Eligible dyads were visited at home by social workers. Caregiver burden was assessed with Mini-Zarit and frailty status with FiND (Frail NonDisabled).

Results . 876 dyads were eligible for PSS. Old persons were $82.2 \pm 5.8$ years old and $77.6 \%$ were women. Most caregivers were women (64.5\%). Most caregivers were the children of the old person (61\%), the rest were mostly spouses. Nearly $64 \%$ of old persons were frail and $38 \%$ were highly dependent. Follow-up was conducted for 686 dyads (78.3\%). Of these, only 569 had PSS. The PSS was mainly for housework and meal preparation. At the time of follow-up, $53 \%$ of the caregivers for whom the old person had PSS had less difficulty helping their old person. Two-thirds (61.5\%) of caregivers were fully satisfied with the PSS. Whether or not they had received the PSS, 73\% of caregivers had reduced burden at 6 months, only $6 \%$ still had a high burden, $17 \%$ were less frail, and $18 \%$ felt healthier than at the time of inclusion.

Conclusions . Our study highlights that non-dependent old person's caregivers also experience burden. Implementing social support for activities of daily living had a major impact on their burden but not on their level of frailty. This means that the determinants of caregiver frailty are more complex and further studies are needed. Keywords: Caregiver's burden. Old person. Personalized social support .

\section{Background}

In the coming years, the population of old persons will increase considerably. In France, the number of persons aged 75 and over will double between 2013 and 2060, to reach nearly 13 million (17\% of the general population) (1). In this context, promoting quality of life, well-being and dignity is a challenge, especially for the most dependent old persons (2). Recent data have shown that about $20 \%$ of the European population over 65 years of age is to some extent dependent and, for the most part, assisted by a caregiver: mainly women family members (3). These caregivers are essential to relay care for the old person and will be increasingly involved in the coming years (4). The economic value of this unpaid informal care was estimated to be between 6.1 and 8.3 billion euros per year in France (5).

In France, between 8 and 11 million persons aged 16 years or more regularly help one or more persons in their entourage for health or disability reasons (i.e. nearly one out of six persons in this age group) (6). In 2015, 3 million persons aged 60 and over and living at home regularly received assistance for a health problem or disability (7). The caregiver's profile varies according to the age of the person being cared for. 
With advancing age, the massive help provided by spouses ( $80 \%$ when assisted persons live in a couple) decreases in favor of help provided by children: $55 \%$ of old persons aged 70 to 74 receive help from their spouse and $32 \%$ from their children. From the age of 75 , this situation is reversed: $32 \%$ receive help from their spouses and $52 \%$ from their children (6).

The type of care provided by caregivers varies greatly, from treatment and care management to shopping assistance and driving out-of-town. When the help provided exceeds the physical and mental capacities of the caregiver, it becomes a chronic stressor generating what is called caregiver's burden (3).

For health workers, it is important to correctly identify the main caregiver, assess the level of stress associated with caregiving, and create a partnership with the caregiver (8). Chronic stress has a negative effect on the caregiver's mental and perceived health (depression, anxiety, psychosomatic and immunological disorders, cardiovascular problems...) and increases the risk of chronic health problems (heart disease, high blood pressure...) and the development of new diseases. In addition, the stress and emotional distress of caregivers has serious consequences for them, in particular their progressive inability to fulfill their role as caregivers, resulting in early institutionalization or unplanned hospitalization of the old person (9) and the risk of abuse or mistreatment for the care recipient, particularly those with dementia and or disruptive behavioral symptoms (10-14). Because of these consequences for caregivers and at a second level for the care recipient, it is necessary to introduce interventions and support for the caregiver (3,15-17).

Two main factors contribute to reducing the burden of primary caregivers. The first is to receive specific help from another family member (18). The second is to benefit from assistance of formal systems (6). In France, the Caisse d'Assurance Retraite et de Santé Au Travail (CARSAT: national administration for retirement and heath at work) is responsible for pension management and health and safety at work, and provides assistance to vulnerable persons. This national institution is represented in all French regions. Southern CARSAT (for the Provence Alpes Côte d'Azur and Corsica regions) has implemented Personalized Social Support (PSS), a formal service developed to provide social support to retired beneficiaries who are not yet dependent but who are at risk of becoming so (19). After assessment by a social worker, support is proposed to old persons who request it, who are not dependent (Group Iso Resources 5 and 6 of the AGGIR grid (20)), and who do not receive other assistance for dependency. However, CARSAT does not fully cover the costs associated with the support: the level of CARSAT coverage is correlated with the income level of the beneficiary. The higher the income, the lower the share of CARSAT coverage. Thus, the financial contributions of old persons vary from $10 \%$ to $73 \%$ of the PSS cost. The impact of this PSS has never been assessed for the beneficiary or for the caregiver.

The main objective was to analyze the impact of PSS implemented for old persons on the burden, perceived health, and frailty of their caregiver. The second objective was to describe the main characteristics of PSS.

\section{Methods}




\section{$1.1 \quad$ Type of study}

A non-interventional observational longitudinal study was conducted in southeastern France in the PACA region (5 million inhabitants, 1 million persons aged 65 years and over) between 2016 and 2017 among 876 dyads: persons aged 70 and over, CARSAT beneficiaries, and their caregivers.

\subsection{Study population}

All beneficiaries were non-dependent, without serious chronic illness, living at home, who applied for CARSAT Southeast assistance, and whose self-appointed caregiver was eligible. After information and consent, the dyads (old person and identified caregiver) who agreed to participate in the study were consecutively included between 1 April 2016 and 30 June 2017 and were followed for 6 months (T6). If a follow-up after 6 months was not possible, the dyad was excluded from the study.

\subsection{Data collection procedure}

At the time of inclusion (T0), the old person was assessed at home by a social worker, according to the usual process, during 60 minutes. Social workers had been trained in the concept of frailty and the modalities of the study. The caregiver was invited to be present at the time of assessment of the old person and to complete a self-assessment questionnaire with the help of the social worker if necessary. When not present, the caregiver was assessed during a phone call by a trained Clinical Research Associate recruited specifically for the study. The 6-month follow-up (T6) was done through a telephone call from the Clinical Research Associate. Dyads were considered "unreachable" after 10 unanswered phone calls. The variables collected are presented in Table 1

\section{$1.4 \quad$. Assessment}

\subsubsection{Mini-Zarit Scale}

There were seven questions on a three-point Likert scale $(9,21)$. The items were scored 0 (never), 0.5 (sometimes), and 1 (almost always). The total score results from the sum of the responses and ranges from 0 to 7 . Four levels of burden were defined:

$-0 \geq$ no burden $\geq 1$

$-1.5 \geq$ light burden $\leq 3$

- $3.5 \geq$ moderate burden $\leq 5$

$-5.5 \geq$ severe burden $\leq 7$ 


\subsubsection{FiND questionnaire}

The FiND questionnaire consists of five questions. Two questions ( $A$ and $B$ ) were specifically aimed at identifying individuals with mobility disability. The difficulty to walk 400 meters $(A)$ or the difficulty to climb 10 stairs (B) revealed the presence of mobility disability. Three additional questions (items C-E) were aimed at assessing signs, symptoms, or conditions commonly considered as components of the frailty syndrome: weight loss (item C), exhaustion (item D), and sedentary behavior (item E). Then the caregiver was classified in three categories: disabled, frail, or robust. Subjects reporting none of these 5 items were classified as robust (22).

\subsubsection{Autonomy Gerontology Iso-Resource Groups (AGGIR)}

This scale is based on the observation of the activities performed or not by the person (20). It measures the level of need for assistance in daily activities. There are six Iso Resources Groups (GIR), from 1 to 6 with decreasing dependency. Group 6 corresponds to independent subjects.

\subsubsection{Frailty Group Iso-Resource Evaluation (FRAGIRE)}

This grid consists of 17 questions (23). The result is calculated according to an algorithm that produces a score from 0 to 100 . This score reflects 3 levels of frailty

- Low frailty: scores $<40$

- Intermediate frailty: $40 \geq$ score $<60$

- High frailty: score $\geq 60$

\subsection{Data analysis}

Statistical analysis was conducted using SPSS Statistics version 20 software. All variables were examined through classical descriptive analysis. Qualitative variables were described by their frequencies and percentages and quantitative variables by their mean and standard deviation $( \pm)$, minimum, median, and maximum.

A univariate analysis was performed to verify that the population at T6 was comparable to the population at T0. The variable "getting help», recorded as yes/no/yes + nursing, was examined by univariate analyses. The associations between qualitative variables were measured by the Chi 2 test and the exact Fischer test for small numbers. A Student Test or Analysis of Variance (ANOVA) was performed for the quantitative variables.

\section{Results}




\subsection{Characteristics of the dyads included in the study}

The study flowchart is presented in Figure 1. Of the 876 dyads interviewed at the time of inclusion, the follow-up was completed for 686 of them (78.3\%). For the other 190 dyads, for which follow-up was not possible, the most frequent reasons were unreachable patient and/or caregivers ( $12.1 \%$ of the cohort), withdrawal $(7.5 \%)$ or death of the old person $(2 \%)$.

The characteristics of the remaining 686 dyads are summarized in Table 2. The population at follow-up had the same characteristics as the total population at the time of inclusion. The average age of the old person was 82.3 years $( \pm 5.9) ; 11 \%$ were over 90 years old. The great majority were women $(78 \%)$ and half lived alone (53\%). The old persons were not dependent and were classified in GiR 5 or 6 , respectively $48 \%$ and $52 \%$. According to the FRAGIRE scale, $94 \%$ were frail; $48 \%$ of them were at high risk of frailty.

In $66 \%$ of cases, caregivers were women with an average age of 62.7 years $( \pm 13.6)$. Most of the time, caregivers were children or stepchildren (62\%); spouses represented $27 \%$. The others $(11 \%)$ were friends, neighbors, grandchildren, and siblings. Among the other caregivers, $71.7 \%$ were family members. In $75 \%$ of the cases, the caregivers were a couple and had children, $35 \%$ of whom were still living with them. Almost half $(48 \%)$ of caregivers had a high level of education and almost $40 \%$ of them were employed. For $48 \%$ of them, incomes were low.

At the time of inclusion, the support provided by caregivers had an impact on their life, particularly on their outings and their availability to have free time for a few days. In addition, $92.5 \%$ of caregivers experienced difficulties in providing support. Many reasons were reported, including poor health, lack of time, and negative impact on their other family obligations (for example, the time available for children). One in five caregivers also reported a lack of material or financial resources. Finally, $17 \%$ of them indicated that they had a poor relationship with their old person.

In addition to CARSAT's support, one in four dyads (27\%) received intervention from another professional, in most cases (88\%) a health professional.

\subsection{Characteristics of the personalized social support \\ (PSS)}

PSS was mainly for household chores (93.6\%); $17.3 \%$ received shopping assistance, and $16.1 \%$ received ironing assistance. Other types of support included escort during outings (6.7\%), meal preparation assistance (6.2\%), grooming assistance (2.3\%), meal assistance (1.9\%), management assistance (1.9\%), and dressing assistance $(0.9 \%)$ (Table 3 ).

One in five old persons received two supports, and 10\% more than two. The most frequent help association was household chores and ironing $(29 \%)$ or shopping $(27 \%)$ or meal preparation $(5 \%)$ or outings assistance (5\%). 


\subsection{Level of satisfaction with the PSS}

Most caregivers, as well as the old persons, were very satisfied with the quality of PSS (Table 3). Almost two-thirds of old persons as well as their caregivers felt that their needs were fully met. The main cause of dissatisfaction was the number of hours received, which was considered insufficient by two-thirds of the dyads. One out of five felt that they should have had other support.

Nearly $9 \%$ complained of dysfunctions in the organization of the support plan, such as delays, absences, and frequent changes of service providers. But these problems were resolved quickly in half of the cases (Table 3).

\section{$1.4 \quad$ Refusal of the PSS}

For about one-fifth of dyads, PSS was not implemented at the time of the follow-up. This was mainly due to the old person's refusal to receive someone at home (29.9\%), price considered too high (19.9\%), and proposed support considered not adequate (8.5\%) or could not be provided (6.8\%). In addition, $10 \%$ preferred other support systems than PSS. And 11\% were still awaiting implementation. Finally, $16 \%$ reported having other reasons for refusing PSS, including institutionalization.

The demographic characteristics of the dyads who did not receive PSS were similar to those of the dyads who received it. However, caregivers who did not have PSS had a poorer relationship with their old person and were less satisfied with their relationship with the health professional.

\subsection{Impact of the PSS}

Most dyads received PSS (569 with PSS versus 117 without PSS), which reduced the power of statistical comparisons and thus the ability to prove the impact of the PSS intervention. Among the caregivers who received PSS, a minority $(n=38)$ reported that they had reduced the number of hours they spent with their old person. For more than $80 \%$ of caregivers who received PSS or did not receive it, the time spent with their old person remained unchanged from the inclusion time, averaging $7.5 \pm 7.0$ hours and $3.8 \pm 2.5$ visits per week.

In addition, among caregivers who received support, obtaining support for a specific task was associated with a reduction of the time spent on that task. This was true for the household chores: $74 \%$ of caregivers who provided home help reported that they had reduced the time spent on this task by obtaining specific household help, while the decrease in time spent on household chores was only $22 \%$ for those who had not received specific help. Time reduction was also significant for ironing assistance $(55.6 \%$ vs $29.1 \%, p<$ $0.05)$, outings assistance ( $31.8 \%$ vs $8.7 \%, p<0.05)$, shopping assistance $(56.7 \%$ vs $10.1 \%, p<0.05)$, and meal preparation assistance $(36.4 \%$ vs $8 \%, p<0.05)$ (Figure 2$)$ 
Figure 3 shows the degrees of change observed for burden, perceived difficulties, self-rated health, and frailty at the 6-month follow-up. At follow-up, 53\% of caregivers experienced fewer difficulties in caring for their old person. This improvement was proportional to the support provided: it was significant for those who had a nursing assistance in addition to PSS compared to those who had only PSS and those who had no assistance at all $(62.7 \%$ and $53.6 \%$ respectively vs $39.1 \%, p<0.05)$.

The burden was reduced for $73 \%$ of caregivers, whether or not they received PSS. The analysis of the mini-Zarit items revealed that caregivers who received PSS obtained better results on the item concerning the impact on their daily life ( $6.3 \%$ vs. $11.8 \%$ for those without PSS, $p<0.05)$.

At follow-up, less than one in five caregivers felt their health status was better than at the time of inclusion. In addition, according to the FiND questionnaire, $17 \%$ of them were less frail, but $12 \%$ appeared frailer. The improvement in frailty was not linked to the implementation of PSS, even if the items constituting the FiND were analyzed separately. However, the improvement in frailty was greater for spouses than for other categories of caregivers ( $23 \%$ vs. $16 \%$ for child caregivers and $6 \%$ for other caregivers, $p<0.05)$. Caregivers living with the old persons were more likely to have improved their frailty compared to caregivers not living with them $(24.5 \%$ versus $12.9, p=0.000)$. They were, for the most part, spouses, and therefore older and frailer than other caregivers.

At follow-up, $15 \%$ of the old persons reported better health status, while $31 \%$ reported poorer health. These changes in health status were not related to the implementation of PSS. Concerning the level of caregiver involvement, those caregivers who performed the most tasks ( $>3$ tasks) were more likely to have reduced their level of burden $(90.1 \%$ vs. $84.3 \%, p=.008)$, less likely to have difficulty caring for the old person ( $59 \%$ vs. $42 \%, p=.001)$, and more likely to have improved their level of frailty $(19.2 \%$ vs. $9.4 \%$, $p=.013)$. Caregivers with a moderate/severe burden at inclusion (37.9\%). were more likely to have increased their level of burden $(94.8 \%$ versus $84.2 \%$ who decreased by at least one level or more, $p=$ $0.000)$ and were more likely to have less difficulty caring for their old person $(65.3 \%$ versus $44.4 \%, p=$ $0.000)$.

\section{Discussion}

No studies, to our knowledge, have been conducted on caregivers of a non-dependent old-age population without serious chronic disease living at home. Most studies have focused on caregivers of dependent populations (24). Our results show that the population of caregivers of non-dependent old persons has a profile similar to that of caregivers of dependent old persons $(6,19)$ and persons with disabling illness $(11,25-27)$ in terms of socio-demographic characteristics and of level of burden, which also has a negative impact on their health $(24-26,11)(19,26-28)$. Our results also show that the caregiver's burden appears well in advance of the recipient's dependency. Moreover, the number of hours and tasks carried out are as numerous as those carried out by the caregivers of dependent persons (19).

PSS was implemented for $83 \%$ of old persons. Most dyads were satisfied and considered that it covered their needs. To the extent that almost all participants benefited from PPS, the measurement of impact 
was limited due to the size of the two subgroups (569 with PSS versus 117 without PSS). Paradoxically, results showed that, at follow-up, the burden level had improved for $73 \%$ of caregivers, whether they had PSS or had merely been consulted about needing PSS.

According to a literature review, the burden includes several component: a psychological component related to the difficulties experienced and an emotional component related to the relationship with the old person, and finally a physical component related to the type and number of tasks performed (29). That the burden improved for all caregivers suggests that the consultation of social workers with the caregivers to identify the needs of the old person for the implementation of PSS had a positive impact on two of the three components of burden, the psychological and emotional components. Indeed, the consultation could be felt by caregivers as a space to talk, a time to be listened to, which allowed them to express their difficulties and which reduced their feeling of loneliness. This consultation probably reassured them, and they were able to project themselves towards the possibility of obtaining future support if it became necessary (30).

Low social support or social isolation, and bad relationship between caregiver and the care recipients are known to increase the risk of burden $(19,31)$ and abuse in community-dwelling old persons. Social support is a protective factor against abuse of old patients (32). This study did not aim at assessing mistreatment or abuse of the old persons. Yet we can hypothesize that this evaluation for PSS, accepted or not, prevented some mistreatment, abuse, or early unplanned hospitalizations particularly for caregivers at high risk, i.e, frail, high burden and high number of daily tasks. (13).

The perceived health and the frailty level improved only for one in five caregivers; this was unrelated to obtaining PSS (33). The fact that the burden, perceived health, and frailty improved independently of the implementation of PSS shows that the impact of a dedicated consultation is very positive overall, as shown in the bibliography (34).

As expected, obtaining PSS reduced the amount of time the caregiver spent performing the tasks. The results show that obtaining specific help reduces not only the time spent on the targeted task but also the time spent on other tasks. This is particularly true for caregivers who were ironing or cleaning before obtaining PSS; many (30\% and $20 \%$ respectively) reported reducing the time spent on this task, even if the assistance provided by PSS was not specific to the task. It is likely that the professionals involved did more than the targeted service and that the old persons were able to focus on fewer tasks but more effectively, which indirectly relieves the caregiver. This could also explain the improvement in the difficulties of caregivers, which was correlated with the implementation of PSS and affected $56 \%$ of caregivers with PSS compared to $39 \%$ of those without it $(p<0.05)$.

However, except in 38 cases, the number of hours and weekly visits to the old person did not decrease since obtaining the PSS. Several explanations are possible. First, data were collected only for caregivers who did not live with the old person, which may have biased the positive impact of the PSS. We found that spouses were the caregivers who did the most work, but the number of hours was not collected for them, so it is possible that the effect of PSS may not be highlighted. Second, the needs may have 
increased during the 6 months or they were not fully covered by CARSAT, and therefore the impact on the amount of time may be moderated. Finally, maintaining the number of hours devoted to assistance may not have the same qualitative significance. Thanks to PSS, the caregiver spends less time performing domestic tasks but spends more time on the relationship with the old person.

Among the biases in selection, the first is related to the financial contribution requested from the old person. Our population is precarious: the number of caregivers close to the poverty line is much higher than that of the general population of the PACA region: $17 \%$ in PACA against $49.9 \%$ for our study (35). However, some caregivers may have underestimated their incomes because they feared that CARSAT would refuse the social support requested or that the contribution requested would be too high. A second potential selection bias is that we included old persons who asked for help with daily living tasks. Thus, the status of the old persons included in our study is in an intermediate level of autonomy. Our results showed that only $5 \%$ of the old persons were assessed as at low risk of frailty according to the FRAGIRE grid. A third limitation regarding the measurement bias on the health status of caregivers is that we did not collect objective health elements, such as walking speed. As a result, comparisons of pre- and postPSS health status were based solely on perceived health.

The originality of this study is its target: caregivers of old persons without serious chronic diseases, autonomous, and living at home. No studies, to our knowledge, have targeted these caregivers, although in terms of frequency, they are certainly the most numerous and constitute a real public health challenge, especially in the future.

Moreover, while most dependency studies have focused on psychological or medical support activities, our study concerns the impact of social assistance exclusively. The study is based on a large sample and a small proportion of persons lost to follow-up. All these points constitute a good level of internal validity.

These results seem to underline the importance of supporting natural caregivers, and the benefit of a dedicated consultation. These consultations should target, first of all, caregivers who have many tasks and/or a high level of burden.

There are many organizations founded and managed by informal caregivers, whose purpose is to provide information and support to peers (6). Unfortunately, they are not always easily accessible. They require that caregivers be aware of their role as caregivers, and that they have sufficient awareness to detect not only the needs of the person they support, but also their own needs and/or difficulties. In addition, these organizations are often focused on a particular pathology, which may exclude caregivers of old persons with no particular pathology.

The first action would therefore be to systematically identify during routine medical consultations whether a person is a caregiver in order to advise and refer them to dedicated organizations and associations. Action should be taken before physical and/or psychological disorders appear. We could consider setting up a specific consultation for them, as is done in the context of dependent old persons. Most of these types of consultations are medicalized or for psychological care. We believe that these 
consultations could first identify the needs of caregivers and make a situational diagnosis that would make it possible to offer personalized support.

\section{Conclusion}

Our results show that the population of caregivers of non-dependent old persons is similar to that of caregivers of dependent old persons or persons suffering from serious diseases in terms of sociodemographic characteristics but also in terms of burden level, perceived health status, and risk of frailty. The evaluation of the implementation of a social assistance plan to improve home support for old persons shows that the intervention of a social worker in the home, whether or not the assistance plan is officially granted, improves the burden on caregivers, but does not quantitatively change the caregiver's investment in the old person.

Health professionals and/or social workers should identify caregivers in order to offer care adapted to their needs and expectations and not only through the needs of the person being cared for. The implementation of dedicated consultations could help caregivers discover the limits of their role and to evaluate the difficulties they encounter so that they can be supported.

\section{Abbreviations}

PSS: Personalized Social Support

CARSAT: Caisse d'Assurance Retraite et de Santé Au Travail

PACA: Provence Alpes Côte d'Azur

CNIL: Commission Nationale de l'Informatique et des Libertés

CRA: Clinical Research Associate

AGGIR: Autonomy Gerontology Iso-Resource Groups

GIR: Iso Resources Groups

ANOVA: Analysis of Variance

FRAGIRE: Frailty Group Iso-Resource Evaluation

\section{Declarations}

\subsection{Ethics approval and consent to participate}

This study was done in accordance with the bioethics laws at the time of data collection and did not require submission to the Protection of Persons Committee. The study was conducted in compliance with 
the IRB. The approach with the study population was consistent with CARSAT's routine professional practice.

Only data collection concerning caregivers was carried out specifically for this study. An information letter was sent to the dyads and a "non opposition" to the study was routinely collected. All nominative data were managed exclusively by CARSAT, which is authorized by the Commission Nationale de I'Informatique et des Libertés (CNIL) (n2005-38) to deal with the protection of personal data.

\subsection{Consent for publication}

Not applicable.

\subsection{Availability of data and material}

The datasets used and/or analyzed during the current study are available from the corresponding author on reasonable request.

\section{$1.4 \quad$ Competing interests}

The authors declare that they have no competing interests

\section{$1.5 \quad$ Funding}

This study was funded by CARSAT Southeast, as part of the implementation of social support for autonomous old persons. The funder (CARSAT) agreed with the design of the study. Health worker of the CARSAT partly participated to the data collection (old subjects and caregivers at inclusion) on a routine practice. The CARSAT did not participate to the data analysis and interpretation, neither to the writing of the manuscript.

\subsection{Authors' contributions}

ACD, SBG and SG developed the protocol. SA and ACD participated in the data collection. SA and SG were responsible for data analysis. SA, SBG and SG interpreted the data. All authors contributed to the writing and revision and approved the final manuscript.

\subsection{Acknowledgements}


We thank CARSAT and its professionals, in particular Sophie Denicolai and Karine Givone. Thanks to Gary Burkhart for proofreading. Also thanks to the social workers for their hard work.

\section{References}

1. Blanpain, N., Chardon, O. Projections de population à I'horizon 2060 - Institut National de la Statistique et des Etudes Economiques - Insee Premièr. 2010 Oct: Report No.: 1103: 4 p.

2. De Almeida Mello J, Van Durme T, Macq J, Declercq A. Interventions to delay institutionalization of frail older persons: design of a longitudinal study in the home care setting. BMC Public Health. 2012 Aug 6;12(1):615.

3. Carretero S, Garcés J, Ródenas F. Evaluation of the home help service and its impact on the informal caregiver's burden of dependent elders. Int J Geriatr Psychiatry. 2007 Aug;22(8):738-49.

4. Dukhovnov D, Zagheni E. Who takes care of whom in the U.S.? Evidence from matrices of time transfers by age and sex. Popul Dev Rev. 2015 Jun;41(2):183-206.

5. Davin B, Paraponaris A, Verger P. Demographic and socioeconomic factors associated with needs for home assistance among community-dwelling elderly: a study from the French Home Survey Handicaps-Disabilities-Dependence. Rev Epidemiol Sante Publique. 2005 Nov;53(5):509-24.

6. Giraud, O., Outin, J-L., Rist, B., Le Cocq-Foltz, C., Ferrand, A., Kovess-Masféty, V., et al. Revue française des affaires sociales 2019/1: 248 p. m

7. Brunel $\mathrm{M}$, Latourelle $\mathrm{J}$, Zakri $\mathrm{M}$. Un senior à domicile sur cinq aidé régulièrement pour les tâches du quotidien. 2019 Feb. Report No.: 1103: 6 p.

8. Schumacher K, Beck CA, Marren JM. FAMILY CAREGIVERS: caring for older adults, working with their families. Am J Nurs. 2006 Aug;106(8):40-9; quiz 50.

9. Bonin-Guillaume S, Durand A-C, Yahi F, Curiel-Berruyer M, Lacroix O, Cretel E, et al. Predictive factors for early unplanned rehospitalization of older adults after an ED visit: role of the caregiver burden. Aging Clin Exp Res. 2015 Dec;27(6):883-91.

10. Yang $X$, Hao Y, George SM, Wang L. Factors associated with health-related quality of life among Chinese caregivers of the older adults living in the community: a cross-sectional study. Health Qual Life Outcomes. 2012 Nov 27;10:143.

11. Basu R, Hochhalter AK, Stevens AB. The Impact of the REACH II Intervention on Caregivers' Perceived Health. J Appl Gerontol. 2015 Aug;34(5):590-608.

12. McCann JJ, Hebert LE, Bienias JL, Morris MC, Evans DA. Predictors of beginning and ending caregiving during a 3-year period in a biracial community population of older adults. Am J Public Health. 2004 Oct;94(10):1800-6.

13. Orfila F, Coma-Solé M, Cabanas M, Cegri-Lombardo F, Moleras-Serra A, Pujol-Ribera E. Family caregiver mistreatment of the elderly: prevalence of risk and associated factors. BMC Public Health. 2018 Jan 22;18(1):167. 
14. Rivera-Navarro J, Sepúlveda R, Contador I, Fernández-Calvo B, Ramos F, Tola-Arribas MÁ, et al. Detection of maltreatment of people with dementia in Spain: usefulness of the Caregiver Abuse Screen (CASE). Eur J Ageing. 2018 Mar 1;15(1):87-99.

15. Oyama Y, Tamiya N, Kashiwagi M, Sato M, Ohwaki K, Yano E. Factors that allow elderly individuals to stay at home with their families using the Japanese long-term care insurance system. Geriatr Gerontol Int. 2013 Jul;13(3):764-73.

16. Davin $B$, Paraponaris $A$, Verger P. L'aide formelle et informelle reçue par les personnes âgées à domicile. Rev DÉpidémiologie Santé Publique. 2008 Oct 1;56(6, Supplement):S357-8.

17. Schulz R, Beach SR. Caregiving as a risk factor for mortality: the Caregiver Health Effects Study. JAMA. 1999 Dec 15;282(23):2215-9.

18. Hopps M, ladeluca L, McDonald M, Makinson GT. The burden of family caregiving in the United States: work productivity, health care resource utilization, and mental health among employed adults. J Multidiscip Healthc. 2017 Dec 6;10:437-44.

19. Arlotto S, Bonin-Guillaume S, Denicolai S, Durand A-C, Gentile S. [Do caregivers of non-dependent elderly have specific characteristics? Study among 876 elderly-caregiver dyads]. Rev Epidemiol Sante Publique. 2019 Sep 5;67(6):403-12.

20. Aguilova L, Sauzéon H, Balland É, Consel C, N'Kaoua B. [AGGIR scale: a contribution to specifying the needs of disabled elders]. Rev Neurol (Paris). 2014 Mar;170(3):216-21.

21. Zarit SH, Reever KE, Bach-Peterson J. Relatives of the impaired elderly: correlates of feelings of burden. The Gerontologist. 1980 Dec;20(6):649-55.

22. Cesari M, Demougeot L, Boccalon H, Guyonnet S, Abellan Van Kan G, Vellas B, et al. A self-reported screening tool for detecting community-dwelling older persons with frailty syndrome in the absence of mobility disability: the FiND questionnaire. PloS One. 2014;9(7):e101745.

23. Vernerey D, Anota A, Vandel P, Paget-Bailly S, Dion M, Bailly V, et al. Development and validation of the FRAGIRE tool for assessment an older person's risk for frailty. BMC Geriatr. $2016 \mathrm{Nov}$ 17;16(1):187.

24. Pinquart M, Sörensen S. Correlates of physical health of informal caregivers: a meta-analysis. J Gerontol B Psychol Sci Soc Sci. 2007 Mar;62(2):P126-137.

25. Basu R, Hochhalter A, Stevens A. PS1-02: Differential Impact of Caregiving Satisfaction and Stress on Changes in Caregivers Self-Reported Health Status: Findings from REACH-II Intervention. Clin Med Res. 2011 Nov 1;9(3-4):176-176.

26. Elliott AF, Burgio LD, Decoster J. Enhancing caregiver health: findings from the resources for enhancing Alzheimer's caregiver health II intervention. J Am Geriatr Soc. 2010 Jan;58(1):30-7.

27. Belle SH, Burgio L, Burns R, Coon D, Czaja SJ, Gallagher-Thompson D, et al. Enhancing the Quality of Life of Dementia Caregivers from Different Ethnic or Racial Groups. Ann Intern Med. 2006 Nov 21;145(10):727-38.

28. Brodaty H, Green A, Koschera A. Meta-analysis of psychosocial interventions for caregivers of people with dementia. J Am Geriatr Soc. 2003 May;51(5):657-64. 
29. Carretero S, Garcés J, Ródenas F, Sanjosé V. The informal caregiver's burden of dependent people: theory and empirical review. Arch Gerontol Geriatr. 2009 Aug;49(1):74-9.

30. Ahn S, Hochhalter AK, Moudouni DKM, Smith ML, Ory MG. Self-reported physical and mental health of older adults: The roles of caregiving and resources. Maturitas. 2012 Jan;71(1):62-9.

31. Johannesen M., LoGiudice D. Elder abuse: a systematic review of risk factors in community-dwelling elders | Age and Ageing | Oxford Academic. Age Ageing. May2013;42(3):292-8.

32. Serra L., Contador I., Fernandez-Calvo B., Jenaro C., Ruisoto P., Flores N., et al. Resilience and social support as protective factors against abuse of patients with dementia: A study on family caregivers. Int J Geriatr Psychiatry. 2018 Aug;33(8):1132-8.

33. Musich S, Wang SS, Kraemer S, Hawkins K, Wicker E. Caregivers for older adults: Prevalence, characteristics, and health care utilization and expenditures. Geriatr Nur (Lond). 2017 Jan;38(1):916.

34. Dam AEH, de Vugt ME, Klinkenberg IPM, Verhey FRJ, van Boxtel MPJ. A systematic review of social support interventions for caregivers of people with dementia: Are they doing what they promise? Maturitas. 2016 Mar;85:117-30.

35. Joseph C., Rivière S. Provence-Alpes-Côte d'Azur - Plus de 850000 personnes vivent sous le seuil de pauvreté - Institut National de la Statistique et des Etudes Economiques -Insee Flash Provence-AlpesCôte d'Azur - . 2016 Dec. Report No.: 32: p 2.

36. Demaison C., Grivet L., Maury-Duprey D., Mayo-Simbler S. France, portrait social - France, portrait social | Institut National de la Statistique et des Etudes Economiques. 2019: 292 p.

37. Higginson IJ, Gao W, Jackson D, Murray J, Harding R. Short-form Zarit Caregiver Burden Interviews were valid in advanced conditions. J Clin Epidemiol. 2010 May;63(5):535-42.

38. Ribeiro EG, Matozinhos FP, Guimarães G de L, Couto AM do, Azevedo RS, Mendoza IYQ, et al. Selfperceived health and clinical-functional vulnerability of the elderly in Belo Horizonte/Minas Gerais. Rev Bras Enferm. 2018;71(2):860-7.

\section{Tables}

Table 1_ Variables collected from the dyad at the time of inclusion and at follow-up 
Variables collected from caregivers at the time of inclusion (T0)

Sociodemographic data

Age, gender, family status (single/married) and number of children living at home

The relationship with the old person

Spouse caregivers (spouses, partners, and ex-spouses)

Child caregivers (children and stepchildren)

Other caregivers (no family relationship and other family members)

The level of education

(no diploma, Youth training, BTEC First Diploma, A levels, higher education level), which has been grouped into 2 categories:

$<$ A level (UK)

$\geq$ A level

Professional activity grouped into two categories:

Caregivers who are working

Inactive caregivers (includes retired, unemployed and disabled)

Monthly income of the tax household

$<740 €$, between 740 and $1200 €$, between 1201 and $2200 €$, between 2201 and $4400 €,>4400 €$

Income: the variable was created from 3 variables: monthly income, family status, and number of children living at home

low income for single caregivers with incomes below $1200 €$ and caregivers living alone or in couple with dependent children and with incomes below $2200 €(36)$

not low income

Activities performed for the old persons at the time of inclusion

Number of hours and days per week spent with the old person

Number and type of tasks performed with the old person:

administrative tasks, activities of daily living (transportation, household chores, ironing, meal preparation, shopping) and nursing (meal assistance, treatment assistance, getting up, going to bed, dressing, grooming)

\section{The consequences of the assistance provided at the time of inclusion}

Perceived difficulties in fulfilling their role as caregivers (5 points on the Likert scale from "not at all" to "a lot") 
Difficulty in fulfilling their role as caregivers due to their own health status, family obligations, lack of material or financial resources, lack of specialized institutions or services, lack of dialogue with professionals or support services, lack of time, lack of expertise and lack of information.

Feel a negative impact of the help on relationships with other family members, on the day's outings, on leaving for a few days (5-point Likert scale from "never" to "always"). This variable was recorded as binary, "impact or not".

The quality of the caregiver's relationship with the old person (5-point Likert scale from "very good" to "very difficult"). This variable was recorded as binary, "good or bad relationship".

Caregiver Burden: Mini-Zarit Scale (ZBI) $(9,21,37)$

Caregiver's frailty: the FiND questionnaire (22)

Health status and health perceived by the caregiver.(38)

Perceived health measured by a question: "How do you rate your health?" (5 points on the Likert scale from "excellent to "bad")

Variables collected from old persons at the time of inclusion

Sociodemographic data: age, gender, and family status

The level of dependency (20) by the Autonomy Gerontology Iso-Resource Groups (AGGIR) scale

Frailty of the old person (23) by the Frailty Group Iso-Resource Evaluation (FRAGIRE)

\section{Variables collected from caregivers at follow-up}

Number and type of tasks performed with the old person:

management tasks, activities of daily life (transportation, household chores, ironing, meal preparation, shopping) and nursing (meal assistance, treatment assistance, getting up, going to bed, dressing, grooming);

Perceives more difficulties to fulfill their role as caregivers (5-point Likert scale from "not at all" to "a lot")

Impact on perceived difficulties: this variable was calculated from the "perceived difficulties" variable at inclusion and follow-up. It represents the change in perceived difficulties and is divided into 5 levels (from significant improvement to significant deterioration).

Caregiver Burden: Mini-Zarit Scale $(9,21,37)$

Impact on burden: this variable was calculated from the "Mini-Zarit" variable at inclusion and followup. It represents the change in burden level and is divided into 5 levels (from significant improvement to significant deterioration).

Caregiver's frailty: the FiND questionnaire (22)

Impact on frailty: this variable was calculated from the "FiND" variable at inclusion and follow-up. It represents the change in frailty level and is divided into 5 levels (from significant improvement to significant deterioration).

Health status and health perceived by the caregiver. 
Perceived health measured by a question: "How do you rate your health?" (5 points on the Likert scale from "excellent to "bad")

Impact on perceived health: this variable was calculated from the "perceived health" variable at inclusion and follow-up. It represents the change in perceived health and is divided into 5 levels (from significant improvement to significant deterioration).

\section{Variables collected from caregivers and old persons at follow-up}

Receiving help (yes/not)

if receiving help

what help (many possible answers):

household chores

ironing

shopping

transportation

meal preparation

meal assistance

grooming assistance

dressing assistance

securing the house (shower, stairs...)

remote assistance

artistic activities

physical activities

satisfaction (5-point Likert scale), recoded in binary (totally satisfied or not)

if not satisfied, why not?

quality of aid (5-point Likert scale), recoded in binary (very good quality or not) having experienced a problem with the performance of the service which one rapid resolution of the problem 
if not receiving help

reasons for the refusal (economic, change of opinion, proposed aid different from the aid requested...)

Other professional care support (nurse, physiotherapist, ...)

Table 2_ Characteristics of dyads with or without Personalized Social Support (PSS) at inclusion and at follow-up 
Total PSS No PSS $p$

$N=686 \quad n=569 \quad n=117$

Caregiver characteristics

Age. mean \pm SD

$62.7 \pm 13.6 \quad 62.9 \pm 13.3 \quad 61.5 \pm 14.7 \quad .310$

Women \% (n)

$\begin{array}{llll}66(453) & 67(381) & 61.5(72) & .259\end{array}$

Single \% (n)

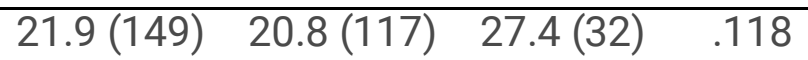

Working \% (n)

$39.8(273) \quad 401(228) \quad 3805(45) \quad .746$

High school education \% (n)

$48.4(326) \quad 49.4(276) \quad 43.9(50) \quad .283$

Low income \% (n)

$48.2(299) \quad 49.5(293) \quad 42.2(46) \quad .166$

Relationship Caregiver - Old Person

\begin{tabular}{|c|c|c|c|}
\hline Spouse caregivers \% (n) & $27.4(188)$ & $27.6(157)$ & $26.5(31)$ \\
\hline Child caregivers \% (n) & $61.5(422)$ & $61.2(348)$ & $63.2(74)$ \\
\hline Other caregivers \% (n) & $11.1(76)$ & $11.2(64)$ & $10.3(12)$ \\
\hline
\end{tabular}

Caregivers limited in the assistance they provided at T0 by

\begin{tabular}{lllll}
\hline own health status \% (n) & $45.1(301)$ & $45.7(253)$ & $42.5(48)$ & .535 \\
\hline family obligations \% (n) & $30.8(205)$ & $31.6(175)$ & $26.5(30)$ & .285 \\
\hline lack of material/financial resources \% (n) & $19(127)$ & $18.1(100)$ & $23.9(27)$ & .149 \\
& & & & \\
\hline lack of specialized institutions/services \% (n) & $7.8(52)$ & $7(39)$ & $11.5(13)$ & .107 \\
\hline lack of dialogue with professionals \% (n) & $6.4(43)$ & $5.6(31)$ & $10.6(12)$ & .048 \\
\hline lack of time \% (n) & $45(300)$ & $44.8(248)$ & $46(52)$ & .807 \\
\hline lack of expertise \% (n) & $15.2(101)$ & $14.1(78)$ & $20.4(23)$ & .091 \\
\hline lack of information about the disease \% (n) & $4.1(27)$ & $3.6(20)$ & $6.2(7)$ & .207
\end{tabular}

Caregivers for whom the assistance provided has had an impact on

\begin{tabular}{lcccc}
\hline family Life \% (n) & $27.3(186)$ & $26.5(150)$ & $31(36)$ & .323 \\
& & & & \\
\hline the day's outings \% (n) & $47.7(324)$ & $47.4(267)$ & $49.1(57)$ & .737 \\
& & & & \\
\hline leaving for a few days \% (n) & $48.1(327)$ & $49(276)$ & $43.6(51)$ & .284
\end{tabular}


\begin{tabular}{lllll}
\hline having a difficult relationship with the old person \% (n) & $17.4(118)$ & $15.9(90)$ & $24.6(28)$ & .026
\end{tabular}

\begin{tabular}{lcccc}
\hline At follow-up & & & & \\
\hline Reduced number of hours per week \% (n) & $55.9(38)$ & $57.9(33)$ & $45.5(5)$ & .447 \\
\hline Fewer difficulties \% (n) & $53(350)$ & $55.7(307)$ & $39.1(43)$ & .001 \\
\hline Burden improvement \% (n) & $73.3(439)$ & $73.5(371)$ & $72.3(68)$ & .821 \\
\hline Self-rated health improvement & $17.7(118)$ & $17.1(95)$ & $20.4(23)$ & .411 \\
\hline Frailty improvement & & & & \\
\hline
\end{tabular}

Table 3_ Characteristics of Personalized Social Support (PSS) and satisfaction 


\begin{tabular}{|c|c|}
\hline SS received \% (n) & \\
\hline Household chores & $96.3(545)$ \\
\hline Shopping assistance & $17.3(98)$ \\
\hline Ironing assistance & $16.1(91)$ \\
\hline Outing assistance & $6.7(38)$ \\
\hline Meal preparation & $6.2(35)$ \\
\hline Grooming assistance & $2.3(13)$ \\
\hline Meal assistance & $1.9(11)$ \\
\hline Management & $1.9(11)$ \\
\hline Dressing assistance & $0.9(5)$ \\
\hline Securing the house & $0.9(5)$ \\
\hline Remote assistance & $0.5(3)$ \\
\hline Artistic activities & $0.5(3)$ \\
\hline Physical activities & $0.2(1)$ \\
\hline Dyads totally satisfied with PSS \% (n) & $61.5(340)$ \\
\hline If not satisfied. Why not? \% (n) & \\
\hline Need more time & $70.5(67)$ \\
\hline Need for other assistance & $20(19)$ \\
\hline Too expensive & $2.1(2)$ \\
\hline Other reasons & $7.4(7)$ \\
\hline Very good quality of PSS\% (n) & $64(355)$ \\
\hline Having experienced a problem with the performance of the service $\%(n)$ & $8.9(48)$ \\
\hline Changes in work hours, delays. absences.... \% (n) & $62.2(28)$ \\
\hline Quality of service. frequent change of service provider.... \% (n) & $22.2(10)$ \\
\hline Other (delay. don't know....) \% (n) & $15.6(7)$ \\
\hline Rapid resolution of the problem \% (n) & $47.2(17)$ \\
\hline
\end{tabular}

\section{Figures}




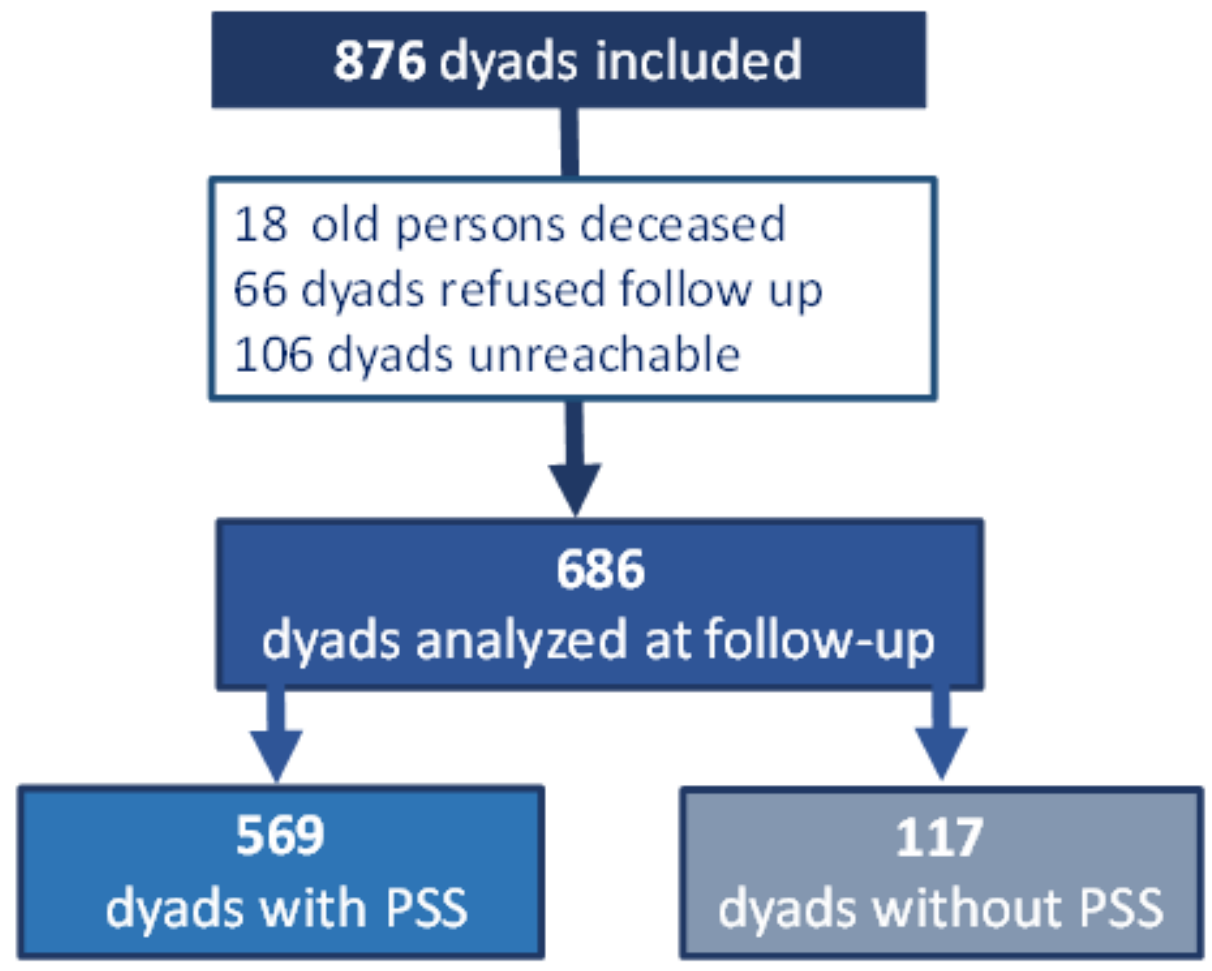

Figure 1

Flow-chart showing participation in the study, from inclusion to follow-up. 


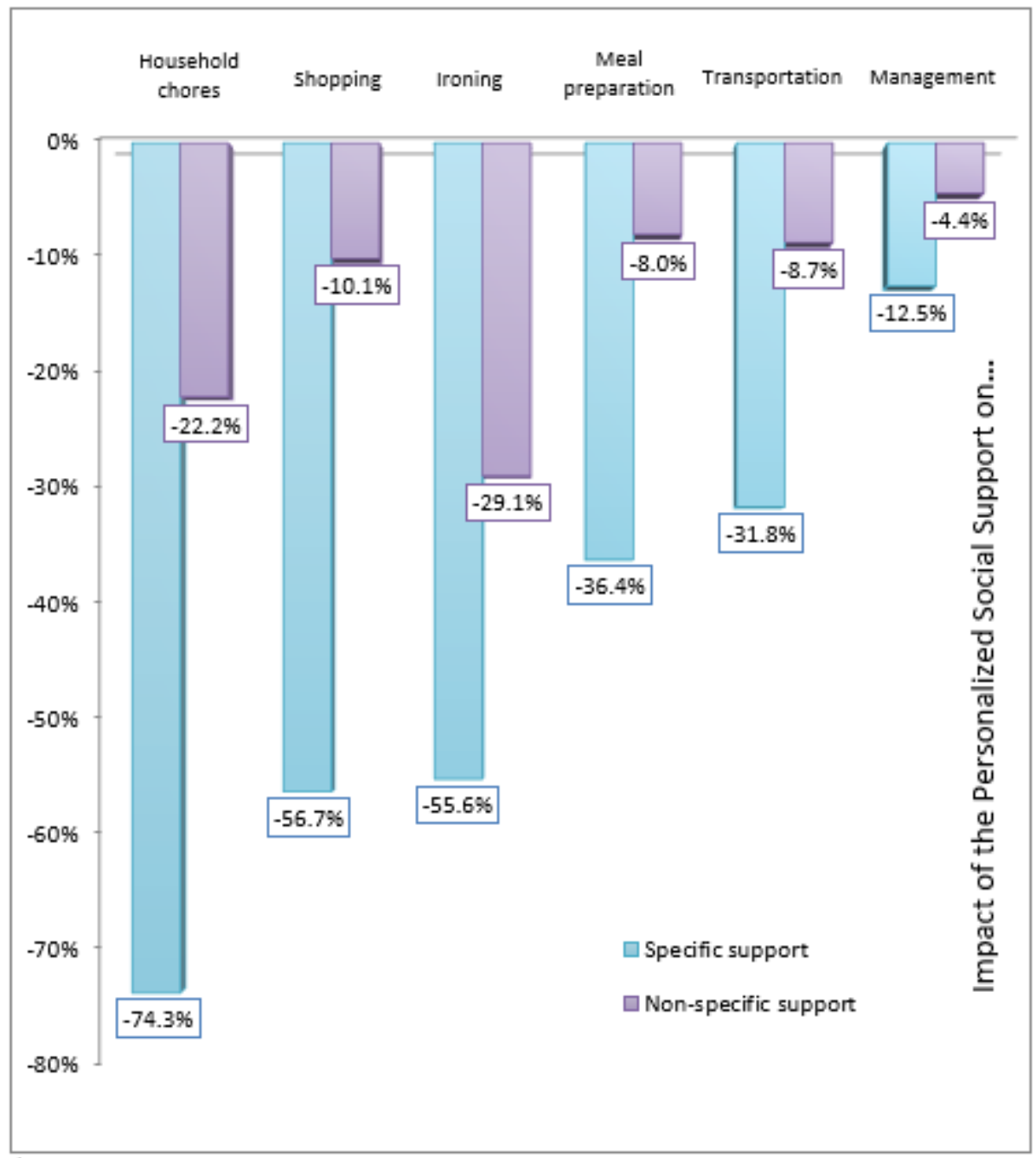

- This image shows how the time spent on a task, such as household chores, decreases as a result of obtaining household-specific assistance (74\%), but also as a result of obtaining assistance other than for the household (22\%).

\section{Figure 2}

Impact of PSS on burden, self-rated health, and frailty. 


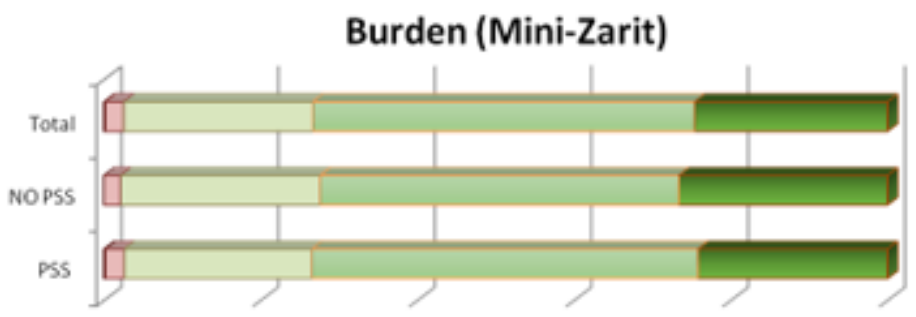

Difficulties

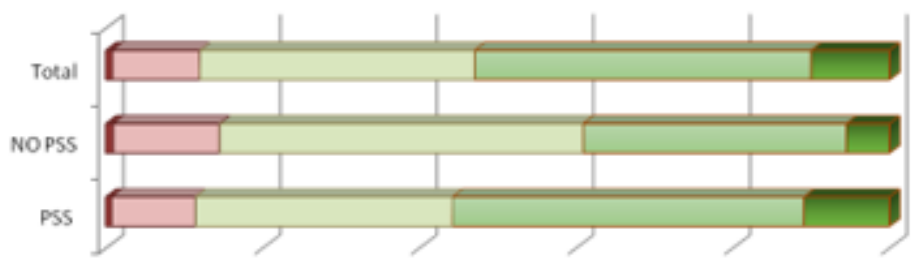

Self-rated health

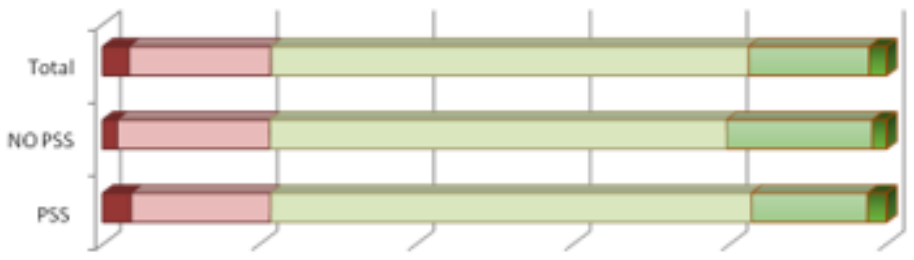

Frailty (FiND)

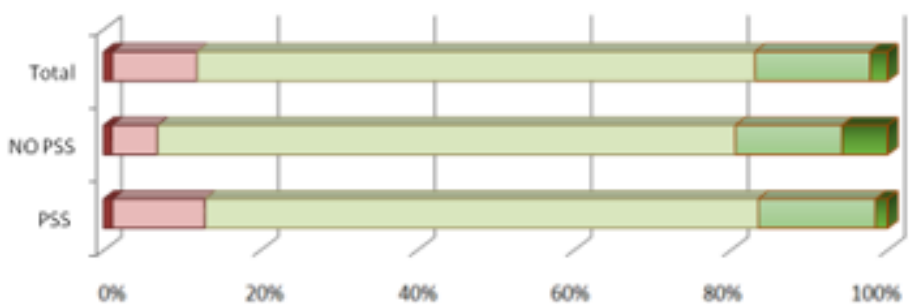

口Significant deterioration $\square$ Slight deterioration $\square$ No change

Elight improvement aSignificant improvement

"PSS = Personalized Social support

\section{Figure 3}

Impact of PSS on the time spent on tasks. 